\title{
Telmisartan prevents the glitazone-induced weight gain without interfering with its insulin-sensitizing properties
}

\author{
Anne Zanchi, ${ }^{1}$ Abdul G. Dulloo, ${ }^{2}$ Christine Perregaux, ${ }^{1}$ Jean-Pierre Montani, ${ }^{2}$ and Michel Burnier ${ }^{1}$ \\ ${ }^{1}$ Division of Nephrology and Department of Medicine, Centre Hospitalier Universitaire Vaudois and University \\ of Lausanne, Lausanne; and ${ }^{2}$ Division of Physiology, University of Fribourg, Fribourg, Switzerland
}

Glitazones are peroxisome proliferator-activated receptor (PPAR)- $\gamma$ agonists with powerful insulinsensitizing properties. They promote the development of metabolically active adipocytes that can lead to a substantial gain in fat mass. Telmisartan is an ANG II type 1 receptor antagonist with partial PPAR- $\gamma$ agonistic properties. Recently, telmisartan has been reported to prevent weight gain and improve insulin sensitivity in diet-induced obese rodents. The goal of this study was to examine the influence of telmisartan on pioglitazone-induced weight gain and insulin-sensitizing properties in the following two models of insulin resistance: a nongenetic model (high-fat-fed Sprague Dawley rats) and the genetically obese falfa Zucker rat. After a 4-wk treatment, the pioglitazoneinduced increase in fat mass was modest in the Sprague Dawley rats and severe in the Zucker rats. In both models, these effects were substantially decreased by concomitant treatment with telmisartan. The effects of telmisartan on body weight and fat mass in the Zucker rats were abolished by pair feeding, suggesting that it is the result of a decrease in food intake. Telmisartan did not interfere with the insulin-sensitizing properties of pioglitazone. This study demonstrates that telmisartan attenuates the glitazone-induced increase in fat mass without interfering with its insulin-sensitizing properties.

glitazones; fat; angiotensin II receptor blocker

GLITAZONES are peroxisome proliferator-activated receptor (PPAR) $-\gamma$ agonists currently used in the treatment of type 2 diabetes (15). These drugs are the most powerful insulin sensitizers available in clinical practice. Their actions are predominantly driven by their influence on adipocyte metabolism. However, because these drugs promote adipogenesis, their uses are often associated with weight gain mainly through an increase in fat mass (6). This side effect may be the reason why some patients stop the drug. The increase in fat mass has been correlated to the efficacy of the drug, since "responders" are among those who show the greatest weight gain (3). Thus the differentiation of preadipocytes to insulin-sensitive adipocytes is a major contributor to the improvement of insulin resistance with glitazones.

Although clinical studies were not able to demonstrate any increase in food intake or decrease in energy expenditure (13), animal studies clearly show that glitazone treatment is associated with an increase in food intake and feed efficiency (7). The mechanisms involved are not known but could be related to the influence of glitazones on neuroendocrine mediators involved in food intake.

Blockers of the renin-angiotensin system have been shown to confer cardiac and renal protection and to lower the incidence of new cases of diabetes in high-risk patients (2). Telmisartan is an ANG II type 1 receptor antagonist that has partial PPAR- $\gamma$ agonistic properties $(1,12)$. For comparison, the $\mathrm{EC}_{50}$ for PPAR- $\gamma$ agonistic effects of telmisartan is of 4.5 $\mu \mathrm{mol} / \mathrm{l}(1)$, and the $\mathrm{IC}_{50}$ for ANG II receptor blockade is of 9.2 $\mathrm{nmol} / \mathrm{l}$ in rats (8). Telmisartan activates PPAR- $\gamma$ in cell-based transient transfection assays by $21-50 \%$ of the maximum level obtained with pioglitazone. Telmisartan has been reported to prevent weight gain and to improve insulin sensitivity in diet-induced obese mice (11) and to attenuate weight gain induced by an energy-dense diet (rich fructose and lard) in Sprague Dawley rats (14). Whether telmisartan, a "partial" PPAR- $\gamma$ agonist, will interfere with the insulin-sensitizing properties of a "full" PPAR- $\gamma$ agonist like pioglitazone is not known. Furthermore, whether telmisartan is able to prevent the weight gain induced by a glitazone in models of insulin resistance, as found previously in models of diet-induced obesity, has not been investigated so far.

The goal of this study was to examine the influence of telmisartan on weight gain induced by pioglitazone in the following two models of insulin resistance: the Sprague Dawley rat fed a high-fat diet rich in lard (a nongenetic model of insulin resistance) and the obese Zucker rat (a genetic model of insulin resistance) known to gain a large amount of body fat when treated with a glitazone (18).

\section{RESEARCH DESIGN AND METHODS}

Sprague Dawley rats fed ad libitum. Male Sprague Dawley rats, age 8 wk were caged singly in a temperature-controlled room $(22 \pm$ $1^{\circ} \mathrm{C}$ ) with a 12:12-h light-dark cycle. They had ad libitum access to water and to a high-fat diet (rich in lard) consisting (by energy) of $45 \%$ fat, $34 \%$ carbohydrates, and $21 \%$ protein (diet 2126; Provimi Kliba, Cossonay, Switzerland). Control $(n=6)$, pioglitazone (20 $\left.\mathrm{mg} \cdot \mathrm{kg}^{-1} \cdot \mathrm{day}^{-1}, n=6\right)$, telmisartan $\left(5 \mathrm{mg} \cdot \mathrm{kg}^{-1} \cdot \mathrm{day}^{-1}, n=6\right)$, and pioglitazone and telmisartan $(n=6)$ were administered for $4 \mathrm{wk}$. Randomization to each group was chosen to have identical average weights in all groups at baseline.

Pioglitazone was incorporated in the diet $(0.24 \mathrm{~g} / \mathrm{kg})$ and telmisartan was added to the drinking water at a concentration of $0.025-0.1$ $\mathrm{g} / \mathrm{l}$ to match an average intake of $5 \mathrm{mg} \cdot \mathrm{kg}^{-1} \cdot \mathrm{day}^{-1}$. At the end of the treatment period, the animals were killed by decapitation for the determination of body composition as described below. Arterial blood was collected for biochemical analysis. 
Obese Zucker rats fed ad libitum. Male obese falfa Zucker rats (Iffa Credo, Lyon, France), aged 8 wk, were fed ad libitum (regular rat chow) with vehicle, pioglitazone, telmisartan, and pioglitazone and telmisartan ( $n=12-14$ in each group) at similar doses as above. Randomization to each group was chosen to have average identical weights in all groups at baseline. They had ad libitum access to water and to a standard chow diet for $4 \mathrm{wk}$ [consisting by energy: $52.8 \%$ carbohydrates, $9.2 \%$ fat, $38 \%$ protein (diet 3200; Kliba)]. A subgroup of rats $(n=$ 6 in each group) was caged singly for accurate food intake and weight control. Another subgroup of animals ( $n=6-8$ in each group) underwent an intravenous glucose tolerance test (IVGTT) at the end of the 4-wk treatment period. They were anesthetized with halothane and had their right femoral artery and vein canulated with a PE-50 catheter. The animals were then returned to their cage. On the following day after an overnight fast, they were placed in a plastic tube for partial restriction of their movements. Intra-arterial blood pressure measurements were performed after $1 \mathrm{~h}$ rest (17). An IVGTT was then performed with a bolus injection of $0.5 \mathrm{~g} / \mathrm{kg}$ glucose and the determination of whole blood glucose and plasma insulin at $0,4,7,10,15$, and $30 \mathrm{~min}$. Insulin resistance index at baseline was assessed by HOMA-IR (9).

Pair-fed obese Zucker rats. Two groups of male obese falfa Zucker rats, aged $8 \mathrm{wk}$, caged singly in a temperature-controlled room, were pair fed on standard chow diet for $4 \mathrm{wk}$ and were treated with pioglitazone $(n=10)$ or pioglitazone and telmisartan $(n=10)$. Randomization to each group was chosen to have identical average weights at baseline. To prevent food spillage, the chow was provided in food pots and in the form of a paste consisting of powdered chow mixed with water (1:1 by weight). Food intake was increased progressively with time to ensure that all groups consumed exactly the same amount of food. At the end of the treatment period, the animals were killed by decapitation for the determination of body composition as described below.
Procedures used in this study were in accordance with principles of animal care and according to the institutional guidelines. The protocol was reviewed and approved by an independent ethical committee for animal research.

Determination of body composition. After death by decapitation, blood was collected; the skull, thorax, and abdominal cavity were incised; and the gut was cleaned of undigested food. The whole carcasses were dried to a constant weight in an oven maintained at $70^{\circ} \mathrm{C}$ and subsequently homogenized. Triplicate samples of the homogenized carcass were analyzed for energy content by bomb calorimetry (10) and for fat content by the Soxhlet extraction method (5). Total body water was calculated from the difference between body weight and carcass dry weight, whereas fat-free-dry mass (a proxy for body protein mass) was calculated from the difference between carcass dry weight and carcass fat (4).

Biochemical parameters. Blood glucose levels were determined with the Glucometer Elite XL (Bayer). Plasma insulin was determined by RIA (Insulin-RIA; Pharmacia, Dübendorf, Switzerland).

Statistical analysis. Data are expressed as means \pm SE. The statistical significance of differences between the treatment groups was evaluated by ANOVA (Minitab) followed by Fisher's test for multiple comparisons. A level of $P<0.05$ was considered statistically significant.

\section{RESULTS}

Sprague Dawley rats fed ad libitum. Gain in body weight was significantly less in telmisartan-treated rats than in controls or in pioglitazone-treated rats [final body weight (g, means \pm $\mathrm{SE}$ ): control, $428 \pm 7$; pioglitazone, $443 \pm 8$; telmisartan, $401 \pm 6$; pioglitazone-telmisartan, $409 \pm 14, P=0.01$ by
Fig. 1. Mean body weight, abdominal fat, and total body fat $(\mathrm{g})$ of control or telmisartan-treated rats $(A)$ and pioglitazone- or pioglitazone- and telmisartan-treated rats $(B)$. Shown are means $\pm \mathrm{SE}$.
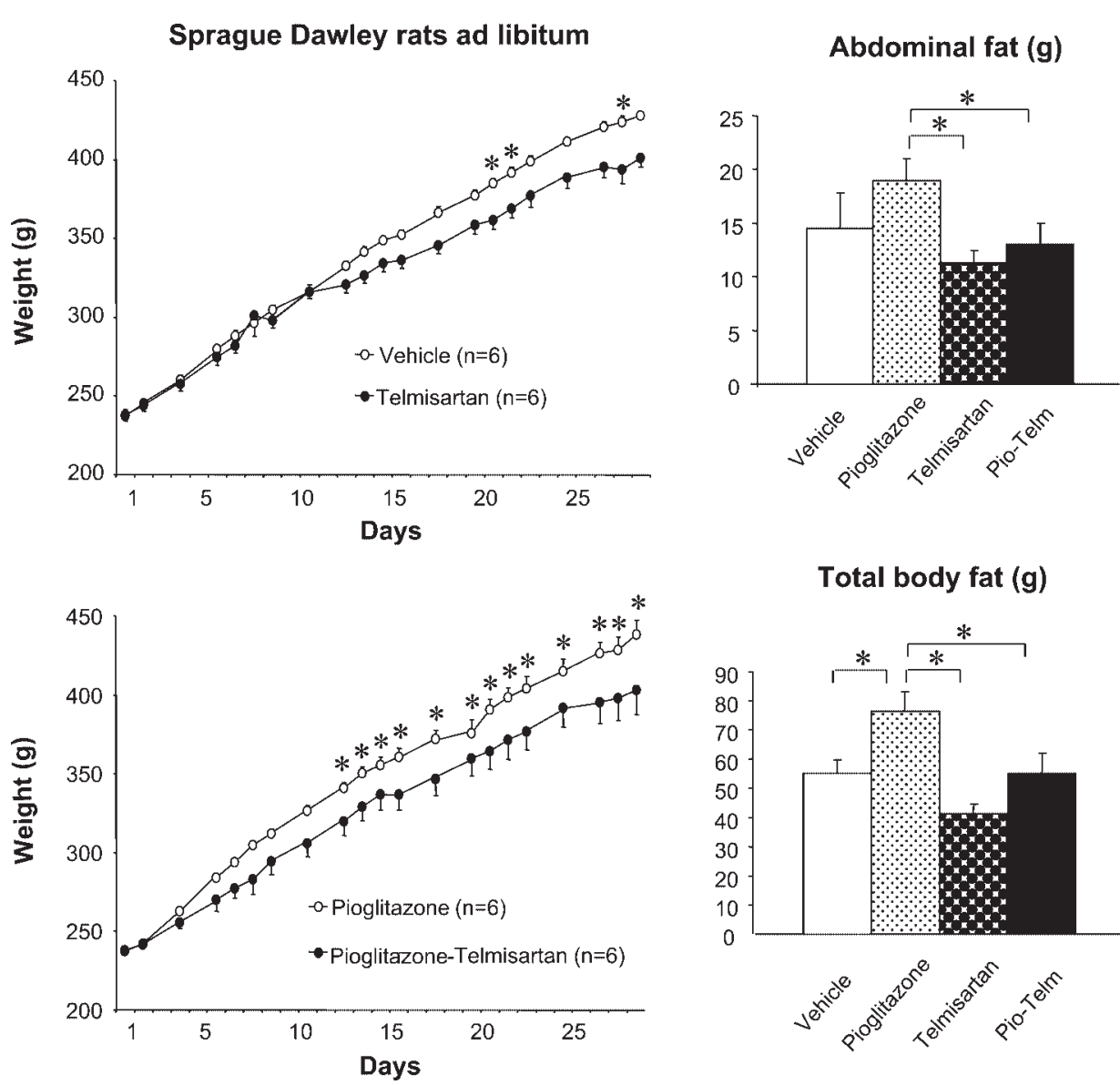

Total body fat $(\mathrm{g})$

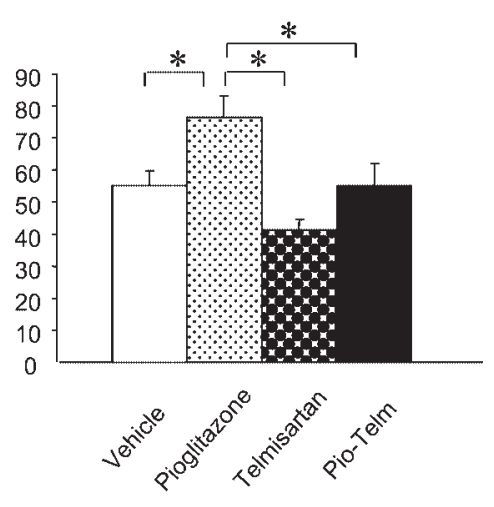


ANOVA; Fig. 1]. Abdominal fat and total body fat increased with pioglitazone and decreased with telmisartan (Fig. 1). Overall, telmisartan added to pioglitazone abolished the increase in body weight and in total body fat because of pioglitazone. During the course of the study, food intake tended to be periodically higher with pioglitazone and periodically lower with telmisartan, with large interanimal variability across time, resulting in no statistical difference for food intake integrated over the 4-wk period [total food intake (g): control, $672.5 \pm$ 9.2; pioglitazone, $706.3 \pm 20.9$; telmisartan, $660.4 \pm 14.9$; pioglitazone-telmisartan, $684.8 \pm 20.7, P=0.3$ ]. Although total food intake over the 4-wk period did not statistically differ between groups, we could demonstrate a strong correlation between the total food intake and total body fat $\left(r^{2}=51.4 \%\right.$, $P=0.002)$. Total body water and fat-free dry mass did not differ between groups. Adding telmisartan to water was not associated with a decrease in water intake.

Insulin levels and the HOMA-IR score were significantly decreased in pioglitazone and pioglitazone-telmisartan treated rats as follows: insulin $(\mathrm{ng} / \mathrm{ml}$, means $\pm \mathrm{SE})$ : control $1.77 \pm$ 0.19 , pioglitazone $0.58 \pm 0.13$, telmisartan $2.04 \pm 0.21$, pioglitazone-telmisartan $0.50 \pm 0.06, P<0.001$; HOMA-IR score: control $17.6 \pm 2.0$, pioglitazone $5.8 \pm 1.4$, telmisartan $20.3 \pm 2.1$, pioglitazone-telmisartan $4.6 \pm 0.6, P<0.001$. Glucose levels did not differ between groups. Telmisartan treatment alone had no significant effect on these parameters.

Obese Zucker rats fed ad libitum: Weight gain, IVGTT, intra-arterial blood pressure measurement. Pioglitazone treatment was associated with a progressive and substantial gain in body weight compared with control rats $(P$ significant from day 4; Fig. 2). Telmisartan treatment significantly attenuated the gain in weight induced by pioglitazone $(P$ significant from day 16). Telmisartan treatment alone attenuated the gain in weight but not significantly. Telmisartan alone had no impact on food intake but with time decreased the hyperphagic effect of pioglitazone treatment. This effect increased week by week to a significant level. The total food intake (g) at week 4 was as follows (in g): control 685.4 \pm 29.2 , pioglitazone $930 \pm 30.5$, telmisartan $676.8 \pm 21.2$, pioglitazone-telmisartan $846.4 \pm$ $18.1, P<0.001$. Total weight gain was strongly correlated to the cumulative food intake $\left(r^{2}=92.9 \%, P<0.001\right.$; Fig. 2$)$. Adding telmisartan to water was not associated with a decrease in water intake.

Plasma insulin levels, the HOMA-IR index, and the insulinglucose response during the IVGTT were significantly improved with pioglitazone (Fig. 3). Telmisartan treatment alone slightly decreased (but nonsignificantly) the basal plasma insulin and glucose levels (the HOMA-IR) and also resulted in a significant reduction in the plasma glucose levels at $0,7,30$ min of the IVGTT. Plasma insulin levels were, however, significantly higher than controls at $10 \mathrm{~min}$ of the IVGTT. In spite of this, the insulin-sensitizing properties of pioglitazone were not affected by telmisartan during the IVGTT.

Intra-arterial mean blood pressure decreased significantly with pioglitazone and telmisartan as follows: control $115 \pm 2$ $\mathrm{mmHg}$, pioglitazone $92 \pm 2 \mathrm{mmHg}$, telmisartan $89 \pm 3$ mmHg, pioglitazone-telmisartan $83 \pm 2 \mathrm{mmHg}(P<0.001)$.

Pair-fed obese Zucker rats: Pioglitazone vs. pioglitazonetelmisartan. The gain in body weight was not different between the pioglitazone-treated group and the pioglitazone- and telmisartan-treated group (Fig. 4). Even when the study was
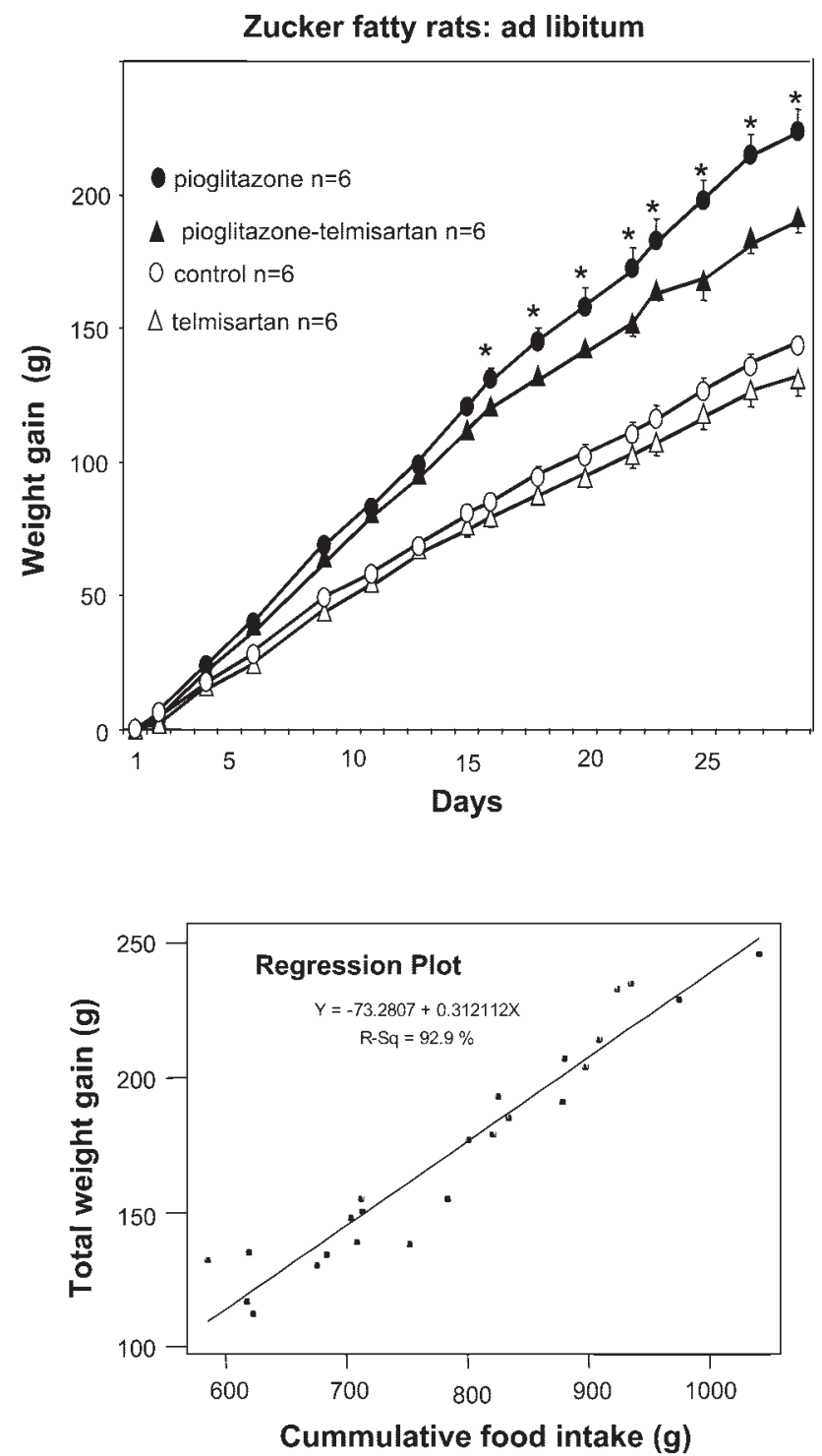

Fig. 2. Weight gain (g) and correlation with cumulative food intake (g) in obese Zucker rats fed ad libitum. $* P<0.05$ vs. pioglitazone- and telmisartantreated rats.

extended to 36 days, there was no difference in weight between groups (means \pm SE: pioglitazone vs. pioglitazone-telmisartan: $539 \pm 4$ vs. $530 \pm 5 \mathrm{~g}$, respectively) nor was there a difference in total abdominal fat $(27.5 \pm 0.7$ vs. $26.2 \pm 0.7 \mathrm{~g}$, respectively) or in whole body fat (316 \pm 6 vs. $320 \pm 4 \mathrm{~g}$, respectively). Telmisartan significantly decreased total water content $(223 \pm 5$ vs. $209 \pm 4 \mathrm{~g}, P<0.05)$ and the heart weight $(1.21 \pm 0.01$ vs. $1.11 \pm 0.01 \mathrm{~g}, P<0.001)$ and had no significant effect on the fat-free dry mass or on the weight of other organs/tissues (kidney, liver).

\section{DISCUSSION}

The main findings of the study presented here can be summarized as follows. 1) The excess gain in body weight and in body fat associated with pioglitazone treatment is modest in a nongenetic (dietary) model and severe in a genetic (defective leptin receptor) model of insulin resistance. 2) In both models, these effects of pioglitazone on excess weight and body fat 


\section{Intra-venous glucose tolerance test}
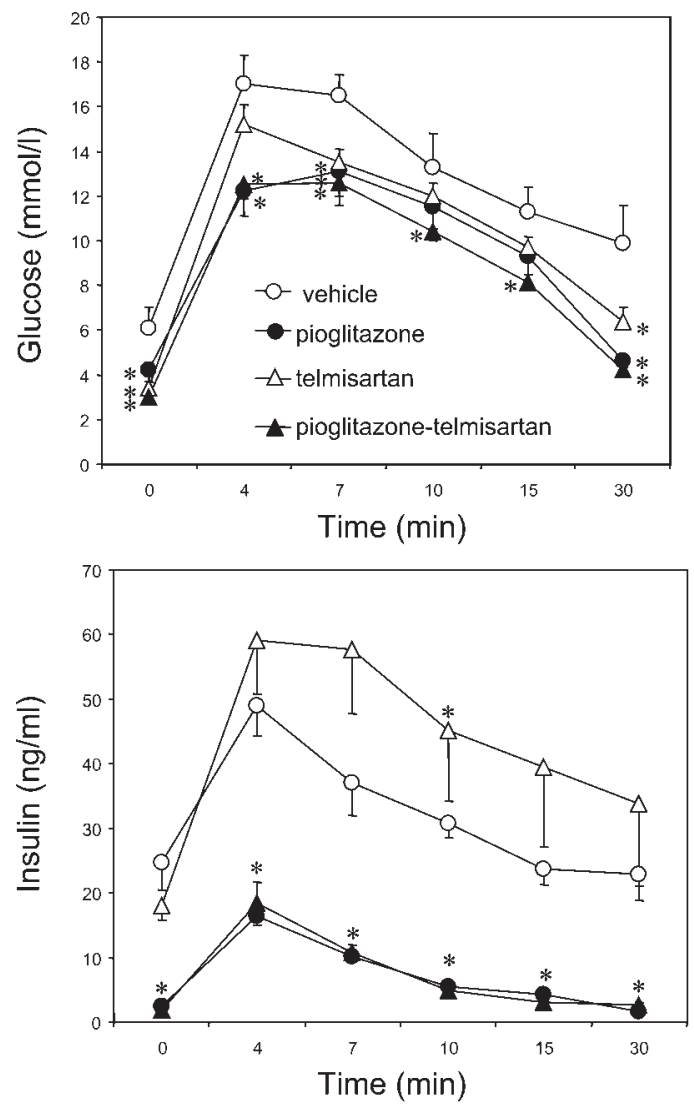

HHOMA=insulin resistance index

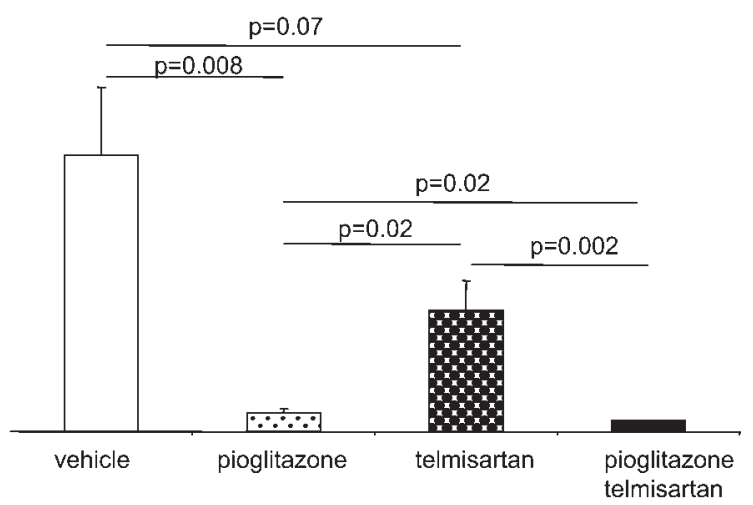

Fig. 3. Intravenous glucose tolerance test and HOMA insulin resistance index in obese Zucker rats. Changes in plasma glucose (mmol/l) and insulin (ng/ml) levels after an iv bolus of $0.5 \mathrm{~g} / \mathrm{kg}$ glucose. ${ }^{*} P<0.05$ vs. control.

gains are attenuated by concomitant treatment with telmisartan. 3) This anti-obesity effect of telmisartan in the obese Zucker rats can be attributed to a decrease in food intake, since cumulative food intake was significantly decreased in rats fed ad libitum, and weight differences were abolished by pairfeeding. Furthermore, telmisartan treatment resulted in lower mean food intake in the Sprague Dawley rats, although no statistical differences could be observed because of the large intragroup variability in total food intake. However, our findings of a strong positive relation between food intake and weight gain in both models is consistent with an important role for reduced food intake in the antiobesity effect of telmisartan. 4) In spite of the described PPAR- $\gamma$ properties of telmisartan, our data suggest that it does not improve the insulin-glucose response to an intravenous glucose bolus in the insulin-resistant obese Zucker rat. However, they also suggest that telmisartan does not interfere with the powerful insulin-sensitizing properties of pioglitazone in this model.

Telmisartan is an ANG II receptor blocker that has PPAR- $\gamma$ properties in vitro independent from its angiotensin type 1 receptor blocking properties. In vivo, telmisartan has been shown to prevent weight gain and to improve insulin sensitivity in a mouse model of diet-induced obesity (11). In Sprague Dawley rats, in which weight gain was induced by a highfructose high-lard diet, telmisartan has also been reported to prevent weight gain, to reduce the accumulation of visceral fat, and to decrease adipocyte size and hepatic triglyceride content (14). In this latter study, the food intake (in absolute terms) was lower in telmisartan-treated rats, and energy expenditure (expressed per unit body wt) was increased. Although the length of our study was much shorter (4 wk vs. 12 wk) we were able to reproduce the favorable effects of telmisartan on weight gain and accumulation of fat in this model. Furthermore, the original finding in our study is that telmisartan is also able to completely abolish the excess weight gain and fat accumulation induced by pioglitazone treatment in Sprague Dawley rats fed a high-fat diet.

However, because the weight gain induced by pioglitazone is modest, we extended these studies to an insulin-resistant model known to dramatically increase its body weight with a glitazone. Telmisartan alone had a very moderate effect on body weight in this model but substantially prevented the increase in body weight induced by pioglitazone and decreased food intake. To investigate an effect of telmisartan on food intake, we clamped the food intake by pair-feeding these rats. The results demonstrate clearly that, when food intake is the same, gain in body weight is no longer different between pioglitazone- and pioglitazone/telmisartan-treated rats. These data therefore suggest that, in the obese Zucker rat model of insulin resistance, telmisartan strongly attenuates the weight gain induced by pioglitazone through an inhibition of food intake and not through increases in energy expenditure. Because adding telmisartan to water was not associated with a

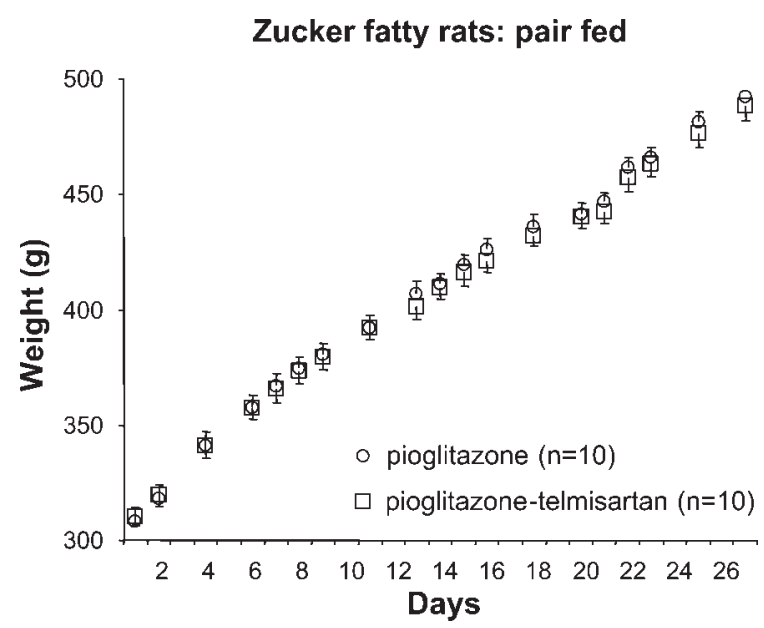

Fig. 4. Mean body weight (g) of pair-fed obese Zucker rats. 
decrease in water intake, a taste aversion of telmisartan in water is an unlikely mechanism for the decreased food intake in telmisartan-treated rats.

Telmisartan treatment did not have a robust effect on the insulin-glucose response in the obese Zucker rats in contrast to earlier findings in the Sprague Dawley rat (1). Nevertheless, and importantly, it did not interfere with the insulin-sensitizing properties of pioglitazone in rats fed ad libitum as may have been feared because of its partial (agonist/antagonist) PPAR- $\gamma$ properties (11). Glucose homeostasis was, however, not studied in pair-fed Zucker rats; thus, these data do not inform on the interaction of telmisartan on the insulin-sensitizing properties of pioglitazone when body weight remains the same.

Weight gain resulting from glitazones is sometimes related to fluid retention due to the activation of renal sodium retention (17). Through its ANG II antagonistic properties, telmisartan could prevent this effect. Indeed, when telmisartan was administered together with pioglitazone, the total water content of the rats showed a tendency to be lower in the Zucker model. However, this small difference is body water does not explain the large differences in body weight found when these rats are fed ad libitum.

Glitazones are PPAR- $\gamma$ agonists with powerful insulinsensitizing properties. Their main actions are related to the development of metabolically active insulin-sensitive adipocytes whose accumulation can, however, lead to a substantial gain in "healthy" fat mass. The fact that telmisartan is able to prevent the glitazone-induced weight gain, without interfering with its insulin-sensitizing properties will motivate future studies, particularly at the neuroendocrine level. Although different mechanisms may be involved, a decrease in food intake with telmisartan is the major contributing mechanism in both animal models treated with pioglitazone. Further studies are required to determine if this favorable interaction with pioglitazone is specific to telmisartan or if it also occurs with other ANG II receptor blockers. In the meantime, since an ANG II receptor antagonist together with a glitazone are often prescribed to diabetic patients, the association of pioglitazone and telmisartan is an attractive combination particularly with respect to weight control.

\section{ACKNOWLEDGMENTS}

We thank Dr. M. Maillard and J. F. Cajot for their contribution to the measurements of plasma insulin.

\section{REFERENCES}

1. Benson SC, Pershadsingh HA, Ho CI, Chittiboyina A, Desai P, Pravenec M, Qi N, Wang J, Avery MA, Kurtz TW. Identification of telmisartan as a unique angiotensin II receptor antagonist with selective PPARgammamodulating activity. Hypertension 43: 993-1002, 2004.

2. Burnier M, Zanchi A. Blockade of the renin-angiotensin-aldosterone system: a key therapeutic strategy to reduce renal and cardiovascular events in patients with diabetes. J Hypertens 24: 11-25, 2006.

3. Chiquette E, Ramirez G, Defronzo R. A meta-analysis comparing the effect of thiazolidinediones on cardiovascular risk factors. Arch Intern Med 164: 2097-2104, 2004.

4. Dulloo AG, Girardier L. Adaptive changes in energy expenditure during refeeding following low-calorie intake: evidence for a specific metabolic component favoring fat storage. Am J Clin Nutr 52: 415-420, 1990.

5. Entenman C. General Procedures for Separating Components of Tissue, edited by Colowick SP and Kaplan O. City, State: Publisher, 1957.

6. Fonseca V. Effect of thiazolidinediones on body weight in patients with diabetes mellitus. Am J Med 115, Suppl 8A: 42S-48S, 2003.

7. Larsen PJ, Jensen PB, Sorensen RV, Larsen LK, Vrang N, Wulff EM, Wassermann K. Differential influences of peroxisome proliferator-activated receptors gamma and alpha on food intake and energy homeostasis. Diabetes 52: 2249-2259, 2003.

8. Maillard MP, Perregaux C, Centeno C, Stangier J, Wienen W, Brunner HR, Burnier M. In vitro and in vivo characterization of the activity of telmisartan: an insurmountable angiotensin II receptor antagonist. J Pharmacol Exp Ther 302: 1089-1095, 2002.

9. Mather K, Hunt A, Steinberg H, Paradisi G, Hook G, Katz A, Quon M, Baron A. Repeatability characteristics of simple indices of insulin resistance: implications for research applications. J Clin Endocrinol Metab 86: 5457-5464, 2001.

10. Miller DS, Payne PR. A ballistic bomb calorimeter. Br J Nutr 13: 501-508, 1959.

11. Schupp M, Clemenz M, Gineste R, Witt H, Janke J, Helleboid S, Hennuyer N, Ruiz P, Unger T, Staels B, Kintscher U. Molecular characterization of new selective peroxisome proliferator-activated receptor $\gamma$ modulators with angiotensin receptor blocking activity. Diabetes 54 : 3442-3452, 2005.

12. Schupp M, Janke J, Clasen R, Unger T, Kintscher U. Angiotensin type 1 receptor blockers induce peroxisome proliferator-activated receptorgamma activity. Circulation 109: 2054-2057, 2004.

13. Smith SR, De Jonge L, Volaufova J, Li Y, Xie H, Bray GA. Effect of pioglitazone on body composition and energy expenditure: a randomized controlled trial. Metabolism 54: 24-32, 2005.

14. Sugimoto K, Qi NR, Kazdova L, Pravenec M, Ogihara T, Kurtz TW. Telmisartan but not valsartan increases caloric expenditure and protects against weight gain and hepatic steatosis. Hypertension 47: 1003-1009, 2006.

56. Yki-Jarvinen H. Thiazolidinediones. N Engl J Med 351: 1106-1118, 2004.

16. Zanchi A, Aubert JF, Brunner HR, Waeber B. Vascular acetylcholine response during chronic NO synthase inhibition: in vivo versus in vitro. Cardiovasc Res 30: 122-129, 1995.

17. Zanchi A, Chiolero A, Maillard M, Nussberger J, Brunner HR, Burnier M. Effects of the peroxisomal proliferator-activated receptorgamma agonist pioglitazone on renal and hormonal responses to salt in healthy men. J Clin Endocrinol Metab 89: 1140-1145, 2004.

18. Zanchi A, Perregaux C, Maillard M, Cefai D, Nussberger J, Burnier M. The PPAR- $\gamma$ agonist pioglitazone modifies the vascular sodiumangiotensin II relationship in insulin-resistant rats. Am J Physiol Endocrinol Metab 291: E1228-E1234, 2006. 